\title{
Relationship between parallel faults and stress field in rock mass
}

\author{
Yuki IMAI $^{1}$, Hitoshi MIKADA ${ }^{1}$, Tada-nori GOTO ${ }^{1}$ and Junichi TAKEKAWA ${ }^{1}$ \\ ${ }^{1}$ Dept. of Civil and Earth Res. Eng., Kyoto University
}

\begin{abstract}
Parallel cracks and faults, caused by earthquakes and crustal deformations, are often observed from regional to laboratory scales. However, the mechanism of the formation of these parallel faults has not been quantitatively clarified, yet. Since the stress field plays a key role in the nucleation of parallel faults, it is fundamentally to investigate the failure and the extension of cracks in a large-scale rock mass (not with a laboratory-scale specimen) due to mechanically loaded stress field. In this study, we developed a numerical simulation code for rock mass failures under different loading conditions, and conducted rock failure experiments using this code. We assumed a numerical rock mass consisting of basalt with a rectangular shape for the model. We also assumed the failure of rock mass in accordance with the Mohr-Coulomb criterion, and the distribution of the initial tensile and compressive strength of rock elements to be the Weibull model. In this study, we use the HPM (Hamiltonian Particle Method), one of the particle methods, to represent large deformation and the destruction of materials. Our simulation results suggest that the compression field have dominant influence for the initiation of parallel faults and their conjugates propagate in uniaxial condition. We conclude that the shearing force would not provoke the propagation of parallel fractures.
\end{abstract}

\section{INTRODUCTION}

Regional-scale parallel faults are often observed, caused by earthquakes or by crustal deformations. These parallel faults or cracks are also observed in laboratory scale. In the area surrounding Japan, one of the typical examples is found in the Izu Peninsula and the offshore area (Figure 1). In these areas, parallel faults are clearly observed. However, the mechanism of formation of parallel faults is not well investigated, and there still remains an important geophysical subject because it is essential for clarifying the system of earthquake occurrence to understand crustal deformation mechanism. If we find the relationship between the formation of parallel faults and stress field applied to rock mass, we could infer stress field loaded to crust from the formation pattern of faults.

The crust around Japan is generally under the compressional condition. Especially the area including the Izu Peninsula suffers the highly compressive force because of the subduction of the Philippine Sea Plate. In addition, the east area of the Izu Peninsula interposed between two left lateral faults: the west one is the Tanna fault and the east one is the WSBF (West Sagami Bay Fracture). Therefore, we assume, in this area, two types of force are loaded: compressive force and shearing force. Furthermore, in this area, not only one direction, conjugate parallel faults are also observed and what form parallel faults in two directions is also unclear.

For interpretation of these crack or fault behaviors, many attempts have been made using fracture mechanics theory. These attempts have successfully represented the propagation of predefined cracks. However, it is difficult to describe the initiation and the coalescence of cracks

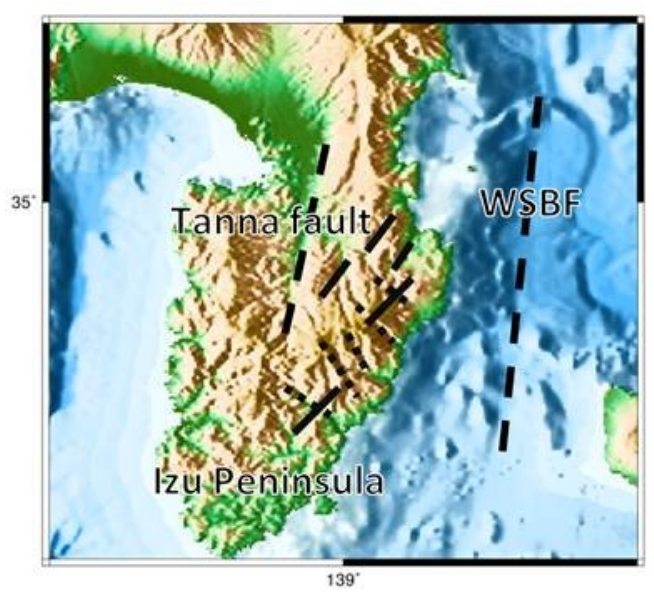

Figure 1 Example of parallel faults in Izu Peninsula. A set of parallel faults in certain direction is shown as solid lines and the conjugate parallel faults are represented as dotted lines. Dashed lines are the main strike slip faults. 
using this theory. Therefore, in the recent years, numerical modeling has been applied to study crack behaviors in rocks ${ }^{1,2)}$. In general, the Finite Difference Method and the Finite Element method are widely used to solve solid deformation problems. However, in these methods, the failure at faults or cracks would not be well simulated when the displacement becomes large or the grid-based structure is broken. On the other hand, the particle methods are free from these difficulties. Thus, we use the $\mathrm{HPM}^{3,4)}$, one of the particle methods, to simulate the formation of parallel faults to investigate the nucleation conditions.

\section{METHOD}

In the HPM, a continuum is represented as a set of particles and a motion of the continuum is approximated as a movement of the particles. Each particle has a weight function calculated by Eq. (1) and the deformation gradient tensor, $\mathbf{F}_{i}$, is calculated using the weighted least squares, minimizing the error function $e_{i}$, in Eq. (2) on each particle:

$$
\begin{aligned}
w_{i j} & = \begin{cases}1-\frac{\left|\mathbf{r}_{i j}^{0}\right|^{2}}{r_{e}^{2}} & \left|\mathbf{r}_{i j}^{0}\right|<r_{e} \\
0 & \left|\mathbf{r}_{i j}^{0}\right| \geq r_{e}\end{cases} \\
e_{i} & =\sum_{j}\left|\mathbf{F}_{i} \mathbf{r}_{i j}^{0}-\mathbf{r}_{i j}\right|^{2} w_{i j}
\end{aligned}
$$

where $\mathbf{r}_{i j}^{0}$ and $\mathbf{r}_{i j}$ are initial and current relative position vector between particle $i$ and $j$ respectively, and $r_{e}$ is the influence radius. Each particle only affected by the particles within the influence radius. The constitutive equation of elastic body is defined as:

$$
\begin{gathered}
\boldsymbol{\varepsilon}_{i}=\frac{1}{2}\left(\mathbf{F}_{i}^{T} \mathbf{F}_{i}-\mathbf{I}\right) \\
\boldsymbol{\sigma}_{i}=2 \mu \boldsymbol{\varepsilon}_{i}+\lambda \operatorname{tr}\left(\boldsymbol{\varepsilon}_{i}\right) \mathbf{I}
\end{gathered}
$$

where $\boldsymbol{\varepsilon}_{i}$ is the Green-Lagrange strain tensor, $\boldsymbol{\sigma}_{i}$ is the Cauchy stress tensor, and $\lambda$ and $\mu$ are the Lame constants. The elastic potential energy, $V$, and the kinetic energy, $K$, are expressed as:

$$
V=\sum_{i} \frac{1}{2} \boldsymbol{\varepsilon}_{i}: \boldsymbol{\sigma}_{i} \Delta A_{i}
$$

$$
K=\sum_{i} \frac{1}{2} m_{i}\left|\mathbf{v}_{i}\right|^{2}
$$

where $\Delta A_{i}, m_{i}$ and $\mathbf{v}_{i}$ are the volume, mass and velocity vector of each particle, respectively. The total energy, Hamiltonian $H$, is calculated as $H=K+V$ and this equation leads the equation of motion of each particle expressed as:

$$
\begin{aligned}
& \rho \frac{\partial \mathbf{v}_{i}}{\partial t}=\sum_{j}\left(\mathbf{F}_{i} \boldsymbol{\sigma}_{i} \mathbf{A}_{i}^{-1} \mathbf{r}_{i j}^{0}+\mathbf{F}_{j} \boldsymbol{\sigma}_{j} \mathbf{A}_{j}^{-1} \mathbf{r}_{i j}^{0}\right) w_{i j} \\
& \mathbf{A}_{i}=\sum_{j} \mathbf{r}_{i j}^{0} \otimes \mathbf{r}_{i j}^{0} w_{i j}
\end{aligned}
$$

where $\rho$ is the bulk density.

In general, rock is a heterogeneous material and this heterogeneity causes failure of rock. Therefore, we consider the Weibull distribution as the basis for heterogeneity. The distribution function of the Weibull distribution is given by:

$$
F(t)=1-\exp \left\{-\left(\frac{t-\gamma}{\eta}\right)^{m}\right\}
$$

where $m$ is the shape parameter describing the scatter of $t, \eta$ is the scale parameter and $\gamma$ is the location parameter, which is the minimum value of $t$. The cohesion and the tensile strength of each particle distribute following this function. In this study, the shape parameter is 3. For both the cohesion and the tensile strength, the scale parameter is $200 \mathrm{MPa}$ and the location parameter is $0 \mathrm{MPa}$.

A lot of shear failure criteria have been proposed for rock. Among these criteria, the Mohr-Coulomb criterion is widely used because of its simplicity. Therefore, we adopt this criterion for the shear failure. The Mohr-Coulomb criterion is expressed by:

$$
\begin{aligned}
& -I_{1} \sin \phi-3 c \cos \phi \\
& +\frac{\sqrt{J_{2}}}{2}\{\sqrt{3}(3+\sin \phi) \cos \theta+3(1-\sin \phi) \sin \theta\}=0 \\
\theta= & \frac{1}{3} \cos ^{-1}\left(-\frac{3 \sqrt{3} J_{3}}{2 J_{2}^{3 / 2}}\right)
\end{aligned}
$$

where $\phi$ is friction angle, $I_{1}$ is the stress invariant, and $J_{2}$ and $J_{3}$ are the deviatoric stress invariants. We calculate this function on each particle. Tensile failure occurs when the tensile stress acting on the rock reaches a critical value, 


\begin{tabular}{|c|c|}
\hline Bulk density & $2800 \mathrm{~kg} / \mathrm{m}^{3}$ \\
\hline Young's modulus & $60 \mathrm{GPa}$ \\
\hline Poisson's ratio & 0.25 \\
\hline Model size & $10 * 20 * 2.5 \mathrm{~km}^{3}$ \\
\hline Particle number & $201 * 401 * 51$ \\
\hline Particle radius & $25 \mathrm{~m}$ \\
\hline Friction angle & 30 degrees \\
\hline
\end{tabular}

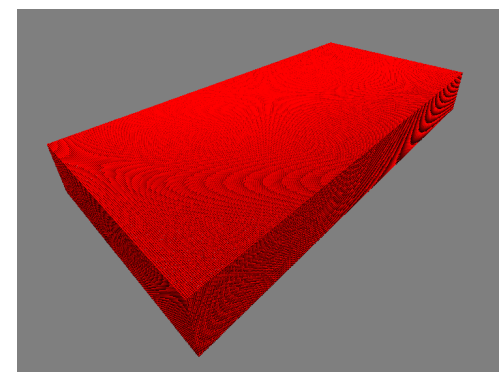

Figure 2 simulation model

which is the tensile strength. This criterion simply expressed as:

$$
\sigma_{t}-\sigma_{1}=0
$$

Table 1 Parameters of the simulation model and compression is positive in this study. After failure, each particle deforms in plastic condition and the stress of each particle is dropped according to magnitude of the maximum principal stress of each particle.

As a simulation model, we assume a rectangular solid (Figure 2) and we also assume that the parameters of the simulation model are almost basaltic. All parameters used in the simulation model are shown in Table 1.

\section{RESULT}

We simulated the rock mass failure in three different loading conditions. Figure 2-4 indicate fractures propagation in middle surface of rectangular calculation models at each loading condition. In all figures, the red plots are the elements have no experience of failure yet and the white plots mean passed failure points, which indicate the elements have previously yield and do not fail currently. The green and blue plots are the active failure points, which are the elements yielding, and the green and blue plots satisfy the shear and tensile criteria respectively.

In the case of only axial compressive force acting, some short fractures appear in middle part of rock (Figure 3(a)) and they grow gradually with loading. Finally, those faults propagate in two conjugate

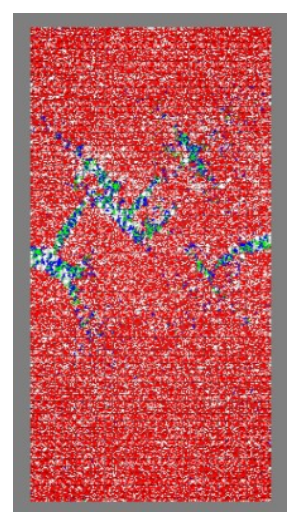

(a)

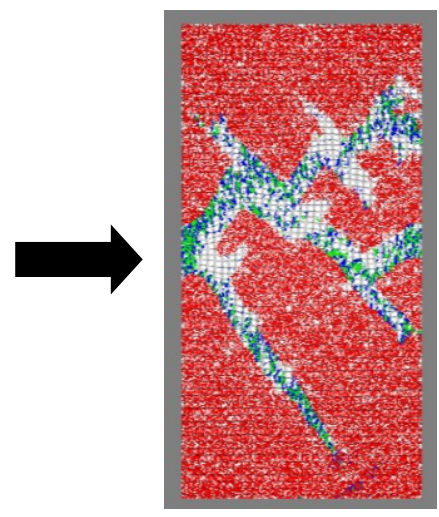

(b)
Figure 3 Faults propagation in uniaxial compression. Some faults are paralleled in two directions.

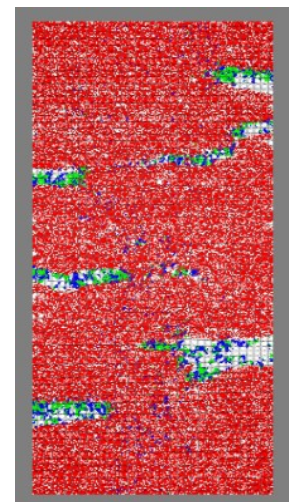

(a)

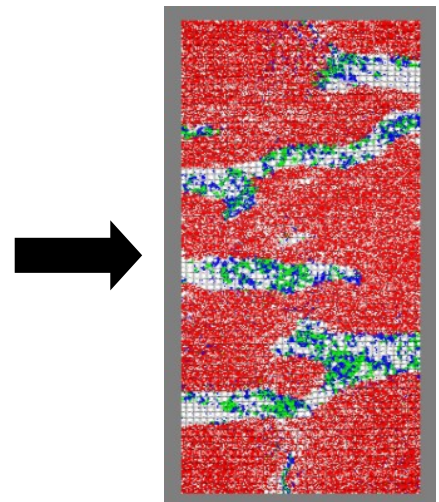

(b)
Figure 4 Faults propagation compressing in biaxial condition. Most fractures are perpendicular to the compression axis.

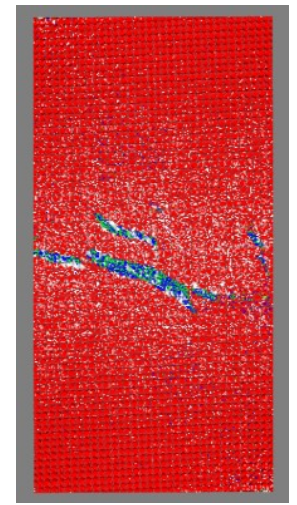

(a)

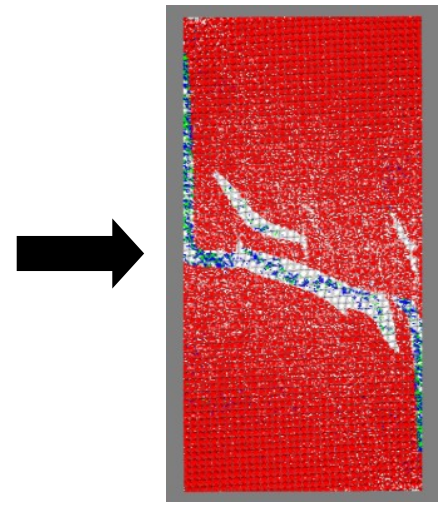

(b)
Figure 5 Faults propagation in the case of shearing forces acing. Only one fault according to the shear forces is mainly active throughout the loading.

directions (Figure 3(b)) and all fractures paralleled in these directions. When the lateral boundaries fixed not to expand in lateral direction in addition to the axial compression, some fractures are generated 
in lateral boundaries (Figure 4(a)) and then they connect each other or propagate to the opposite end (Figure 4(b)). They are parallel in lateral direction and their conjugates do not appear.

Figure 5 shows the calculation results of the case of strike slip force acting on the lateral boundaries. In this case, the lateral boundaries are also fixed not to rotate. Some lined fractures are formed according to the shearing direction (Figure 5(a)) and they combine. Finally, they develop to one large fault (Figure 5(b)).

\section{CONCLUSION}

We simulated the failure of a rock mass in various loading conditions to confirm the factor of the development of parallel fractures and their conjugates. As a result of the simulations, in the case of compressive stress field, the faults are paralleled clearly. When only the axial compressive force is acting, fractures are paralleled and their conjugates are also active as shown in Figure 3. In the case that the expanding of the rock mass in lateral direction is restricted, (i.e. confining pressure is in effect), parallel fractures propagate and they are perpendicular to the axial compression (Figure 4), but their conjugates do not appear. If the strike slip force is added, the direction of fractures propagation is limited by the direction of the shear force and parallel faults are not created (Figure 5). This suggests that the compressive force would be necessary for the initiation of parallel fractures and that the confining pressure would suppress the propagation of conjugate fractures. In Izu peninsula, conjugate faults are obviously observed as shown in Figure 1. Therefore, in this area, compressive force would be dominant and shear forces in the two strike slip faults would not be so efficient.

In this study, we focused only on the initiation of the parallel or conjugate fractures. Therefore, we simulated only changing the type of the force acting on the rock mass without changing the magnitude of the force. The fracture length or interval is considered influenced by the following factors: the magnitude of the forces, the thickness of the crust and the strain rate according to the loading. For the simulation of existing parallel faults such as in Izu Peninsula, we need to conduct.

\section{REFERENCES}

1) Fang, Z., and Harrison, J. P., 2002, Application of a local degradation model to the analysis of brittle fracture of laboratory scale rock specimens under triaxial conditions, Int. J. Rock. Mech. Min. Sci., 39, 443-457.

2) Ma, G. W., Wang, X. J., and Ren, F., 2011,
Numerical simulation of compressive failure of heterogeneous rock-like materials using SPH method, 2011, Int. J. Rock. Mech. Min. Sci., 48, 353-363.

3) Kondo, M., Suzuki, Y., and Koshizuka, S., 2007, Suppressing local oscillation for elastic analysis based on least square approximation using particles, Trans. JSCES, Paper No. 20070031.

4) Kondo, M., Yamada, Y., Sakai, M., and Koshizuka, S., 2010, Development of a particle method for an elastic body considering random packing of powder, J. Soc. Powder Technol., Japan, 47, 531-538. 